localizing value in frontal and temporal lobe epilepsies. Brain Feb 1996;119:1740). (Respond: Dr M Manford, Wessex Neurological Centre, Southampton General Hospital, Tremona Road, Southampton SO16 6YD, UK).

COMMENT. The ILAE classification of seizure types based on electroclinical criteria may be justified for well-defined syndromes, eg benign epilepsy of childhood with centrotemporal spikes, but in the differentiation of frontal and temporal lobe partial epilepsies in adults there is significant clinical/pathological overlap. The authors argue in favor of an anatomicalpathological definition of fronto-temporal seizures, and they disagree with the ILAE classification of 7 different localizable seizure types within the frontal lobes based on electroclinical manifestations. Neuroimaging appears to be the only reliable method of lesion localization for frontal and temporal lobe partial epilepsies.

Magnetoencephalographic analysis of rolandic discharges in benign childhood epilepsy is reported from Kyushi University, Fukuoka, Japan. (Minami T et al. Ann Neurol March 1996;39:326-334). Dipole methods of MEG allow more precise location and quantification of electrically active brain regions compared to the EEG, and the recorded signal reflects intracellular rather than extracellular current flow. Equivalent current dipoles (ECDs) of prominent negative sharp waves of rolandic discharges appeared as tangential dipoles in the rolandic region and showed a limited localization compared with other components. ECDs of preceding small positive waves, positive waves following negative sharp waves, and negative slow waves were located close to negative sharp waves. ECDs of rolandic discharges were localized to the origin of somatosensory evoked magnetic field stimulated at the lower lip on a reconstructed three-dimensional MR image. Rolandic discharges are generated by a similar mechanism to that for the somatosensory evoked responses.

\title{
KETOGENIC DIET MECHANISM OF ACTION
}

The intracellular $\mathrm{pH}$ of the cerebral cortex was studied by the neutral red method in 15 adult rats maintained on ketogenic and control diets for 5-6 weeks. Rats fed the ketogenic diet had more than a 10-fold increase in plasma ketones, but no significant differences in cerebral $\mathrm{pH}$ or in cerebral metabolites and GABA levels were noted. The antiepileptic effect of the ketogenic diet was probably not mediated by cerebral acidosis or changes in cerebral GABA levels. (Al-Mudallal AS, Harik SI et al. Diet-induced ketosis does not cause cerebral acidosis. Epilepsia April 1996;37:258-261). (Reprints: Dr SI Harik, Department of Neurology, University of Arkansas, 4301 W Markham, Slot 500, Little Rock, AR 72205).

COMMENT. The ketogenic diet used in the treatment of some forms of epilepsy in childhood was originally introduced at the Mayo Clinic (Wilder RM. Mayo Clin Bull 1921;2:307), not at Johns Hopkins University, as some recent media publicity would have us believe. Furthermore, some of the earlier work relating to the mechanism of action of the ketogenic diet, not cited in the above paper, also originated at the Mayo Clinic (Millichap JG, Jones JD, Rudis BP. Mechanism of anticonvulsant action of ketogenic diet. Amer I Dis Child 1962;104:506, and 1964;107:593-604). Seizure susceptibility was not modified by a fat diet in normal animals, but an anticonvulsant effect was demonstrated in mice with a seizure threshold lowered by water intoxication and hypoelectrolytemia. In animals and in patients with absence seizures, the 
anticonvulsant effect of the ketogenic diet was unrelated to diuresis, independent of acidosis and ketosis, similar to the effects of acetazolamide, and correlated most closely with a negative balance of sodium and potassium.

\section{ANTICONVULSANT SIDE EFFECTS}

\section{COGNITIVE EFFECTS OF PHENOBARBITAL}

Neurocognitive behavior in 9 children with various epilepsies was evaluated before and at 6 months after discontinuing phenobarbital monotherapy at the Department of Child Neurology, Instituto Nazionale Neurologico "Carlo Besta;" Milano, Italy. The patients had been seizure-free for at least 2 years. All of the scores on the WISC improved and the mean Performance IQ was significantly higher after phenobarbital was withdrawn. Other tests showing significant improvement included the general information subtest on the Verbal IQ picture arrangement on the Performance IQ visual spatial memory, and visual-motoric and attentional skills, as measured by coding and the Trail Making test. (Riva D, Devoti M. Discontinuation of phenobarbital in children: Effects on neurocognitive behavior. Pediatr Neurol 1996;14:36-60). (Respond: Dr Riva, Department of Child Neurology, Instituto Neurologico "Carlo Besta;" 11 Via Celorio, 20133 Milano, Italy).

COMMENT. In this small number of children treated, phenobarbital appeared to have impaired attention, spatial memory, and visual/motor skills. The deficits were reversible and disappeared when phenobarbital was discontinued.

Drowsiness secondary to chronic antiepileptic drug therapy was assessed in 30 patients, using an EEG-based Awake Maintenance Task (AMT) measure, and reported from the Portland Veterans Affairs Medical Center, and Oregon Health Sciences University, Portland, Oregon. (Salinsky MC et al. Epilepsia 1996;37:181-187). Ability to maintain wakefulness was determined during a 6 -min unstimulated trial. One third of AED-treated patients had $>120 \mathrm{~s}$ of drowsiness in contrast to only 1 of 63 controls. Objective EEG drowsiness did not correlate with AED levels or performance measures. Untreated seizure patients had more complaints of lack of vigor despite absence of objective drowsiness on the AMT. Subjective reports of AED-related drowsiness may be unreliable.

\section{LAMOTRIGINE-INDUCED SKIN RASH}

Five of 68 consecutive children treated for epilepsy with lamotrigine developed a skin rash, one a Stevens-Johnson syndrome, in a report from Dalhousie University, and IWK Children's Hospital, Halifax, Nova Scotia, Canada. Two patients required intensive care. The interval between introduction of lamotrigine and the rash varied from 2 to 8 weeks. One child in whom the drug was reintroduced after 6 months had a recurrence of the rash within 30 minutes of a single small dose. In 4 patients taking concomitant therapy, the AEDs were continued during and after the lamotrigine-induced rash. (Dooley J, Camfield $\mathrm{P}$ et al. Lamotrigine-induced rash in children. Neurology Jan 1996;46:240-242). (Respond: Dr Joseph M Dooley, Neurology Division, IWK Children's Hospital, 5850 University Avenue, Halifax, Nova Scotia, Canada B3J 3G9).

COMMENT. Skin rash, especially Stevens-Johnson syndrome, is one of the most disturbing side-effects of AEDs. The introduction of any 